
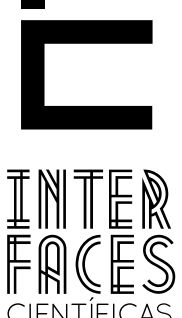

SAÚDE E AMBIENTE

\title{
CONHECIMENTO E INTENÇ̃̃O DE USO DA FITOTERAPIA EM UMA UNIDADE BÁSICA DE SAÚDE
}

Mariana Aparecida Lopes ${ }^{1}$

Adriana Lenita Meyer Albiero
Simoni Obici

\section{RESUMO}

Estima-se que $80 \%$ da população mundial dependa da fitoterapia no que se refere à atenção primária em saúde e grande parte destes tem nas plantas a única fonte de medicamentos. Uma vez que existe uma deficiência no conhecimento dos profissionais prescritores sobre fitoterapia, já que este assunto não faz parte de sua formação acadêmica, surge a necessidade de um plano modificador deste quadro, pelos municípios interessados em ofertar o uso seguro de medicamentos fitoterápicos para sua comunidade. 0 objetivo deste trabalho é identificar o grau de conhecimento e interesse por parte dos prescritores da Unidade Básica de Saúde (UBS) Pinheiros, em relação à utilização de plantas medicinais como forma de tratamento. Foram aplicados questionários a oito médicos e oito enfermeiros da UBS. Os dados foram coletados em novembro de 2010 e depois foram tabulados e analisados. A maioria dos médicos e enfermeiros entrevistados prescreve/indica plantas medicinais eventualmen- te, e as situações de prescrição mais citadas foram: como antidepressivo/calmante, para gripe, tosse e mal estar gástrico. A grande maioria dos entrevistados não teve contato com fitoterapia durante a sua formação e tem interesse em aprender mais sobre plantas medicinais, o que segundo eles poderia aumentar o número de prescrições. A partir destes dados, concluímos que os profissionais não tem muito conhecimento a respeito de plantas medicinais, mas que a maioria deles tem vontade de aprender mais sobre o assunto. Isso é importante, pois estes profissionais poderiam passar mais informações para os usuários a respeito do uso racional e seguro destes medicamentos.

\section{PALAVRAS-CHAVE:}

Medicamentos Fitoterápicos. Plantas Medicinais. Centros de Saúde. 


\section{ABSTRACT}

It is estimated that $80 \%$ of the population depends on the phytotherapy, regarding the primary attention to health, and most of them rely just on the plants as the only source of medicine. Since these professionals do not master the knowledge on phytotherapy prescription, due to the fact that this subject is not part of their academic training, the need for a plan which modifies this framework is necessary, in the municipalities interested in offering safe use of herbal medicines to their community. The objective of this study is to identify the level of knowledge and interest which may be observed among the prescribers of Basic Health Unit (BHU) Pinheiros, regarding the use of medicinal plants as a treatment. Questionnaires were administered to eight doctors and eight nurses from BHU. Data was collected in November 2010 and was then tabulated and analyzed. Most doctors and nurses who were interviewed prescribe/indicate medicinal plants, and, most frequently, as antidepressant/calming, and for flu, coughs and stomach discomfort. The vast majority of respondents had no contact with herbal medicine during their training processes and they were interested in learning more about medicinal plants, so, as they mentioned, the number of prescriptions could be increased. From these data, we conclude that professionals do not have much knowledge about medicinal plants, but most of them want to learn more about it. This is important because these professionals could teach users about the rational and safe use of these drugs.

\section{KEYWORDS}

Phytotherapeutic Drugs. Medicinal Plants. Health Centers.

\section{RESUMEN}

Se estima que el $80 \%$ de la población depende de las hierbas en lo que respecta a su salud primaria y la mayoría de estas plantas es la única fuente de medicamentos. Dado que existe una falta de conocimiento de los prescriptores sobre la medicina a base de hierbas, ya que el tema no es parte de su formación académica, surge la necesidad de la creación de un plan para modificar esta situación en los municipios interesados en ofrecer un uso seguro de los medicamentos herbarios a su comunidad. El objetivo de este estudio es identificar el grado de conocimiento e interés por parte de los prescriptores de la Unidad Básica de Salud (UBS) Pinheiros, relacionado al uso de plantas medicinales como tratamiento. Los cuestionarios fueron administrados a ocho médicos y ocho enfermeras de la UBS. Los datos fueron recogidos en noviembre de 2010 y fueron tabulados y analizados. La mayoría de los médicos y enfermeros entrevistados prescribe / indica las plantas medicinales eventualmente. Las situaciones mencionadas con mayor frecuencia fueron, como antidepresivo / calmante, para gripe, tos y molestias en el estómago. La gran mayoría de los encuestados no tuvo contacto con la medicina herbaria durante su formación y están interesados en aprender más sobre las plantas medicinales, las cuales dicen podrían aumentar el número de recetas. A partir de estos datos, llegamos a la conclusión de que los profesionales no tienen mucho conocimiento sobre plantas medicinales, pero la mayoría de ellos tienen el deseo de aprender más sobre ellas. Esto es importante porque estos profesionales podrían pasar más información a los usuarios sobre el uso racional y seguro de estos medicamentos

\section{PALABRAS CLAVE}

Medicamentos Fitoterápicos. Plantas medicinales. Centros de Salud. 


\section{INTRODUÇ̃̃O}

As plantas são utilizadas há muito tempo como fonte de medicamentos para o tratamento das enfermidades que acometem o homem, de modo a aumentar suas chances de sobrevivência (CARVALHO et al., 2010). Aproximadamente $80 \%$ da população mundial depende da fitoterapia no que se refere à atenção primária em saúde (BRASILEIRO et al., 2008; ORGANIZAÇÃO MUNDIAL DA SAÚDE, 1979; TOMAZZONI et al., 2006; MOREIRA et al., 2002; VEIGA JUNIOR, 2008).

No Brasil, a temática da fitoterapia é bastante abordada e existe constante recomendação da utilização de práticas populares de cura nos serviços de saúde. Várias prefeituras brasileiras têm implementado programas de fitoterapia.

Os profissionais prescritores de modo geral, mas, principalmente os médicos, possuem papel importantíssimo e decisivo na utilização da fitoterapia. 0 que é praticado e propagado por estes profissionais, na grande maioria das vezes, adquire caráter de "lei" e são seguidos pela população. Dessa forma, a opinião e o caráter do profissional médico são decisivos em uma política de fitoterápicos dentro de um serviço de saúde (ROSA et al., 2011).

Mesmo a fitoterapia sendo eficaz, os profissionais de saúde devem orientar as pessoas quanto

\section{MATERIAL E MÉTODOS}

Essa pesquisa foi desenvolvida na cidade de Maringá, situada na Região Noroeste do Estado do Paraná, com uma população de 357.117 habitantes, na UBS Pinheiros, a qual é responsável pelo atendimento de uma área com população aproximada de 30.000 habitantes. Este trabalho é resultado de um projeto desenvolvido por participantes do Programa de Educação para o Trabalho em Saúde (PET-Saúde) da Universidade Estadual de Maringá. ao uso indiscriminado de algumas plantas medicinais. Por ser um assunto de Saúde Pública, caberia aos profissionais de saúde e aos programas nacionais de saúde (Programa Saúde da Família - PSF e Programa Agentes Comunitários de Saúde - PACS) esclarecer as dúvidas da população, orientando a utilização correta de plantas medicinais nas Unidades de Saúde e nas visitas domiciliares. Para isso, o profissional deveria estar devidamente capacitado.

Uma vez que, existe uma deficiência no conhecimento dos profissionais prescritores - médicos, enfermeiros, odontólogos - sobre fitoterapia, já que este assunto não faz parte de sua formação acadêmica, surge a necessidade de um plano modificador deste quadro pelos municípios interessados em ofertar o uso seguro de medicamentos fitoterápicos para sua comunidade (SCHENKEL et al., 1985; VEIGA JUNIOR, 2008).

A partir destes dados, o objetivo deste trabalho é identificar o grau de conhecimento e interesse por parte dos prescritores (médicos e enfermeiros) da Unidade Básica de Saúde (UBS) Pinheiros, em relação à utilização de plantas medicinais como uma forma de tratamento.
Foram aplicados questionários a oito médicos e oito enfermeiros da UBS, o que corresponde a todos os profissionais que trabalhavam na Unidade no período de realização do trabalho. Os participantes assinaram um termo de consentimento, afirmando estarem de acordo com a natureza e finalidade do trabalho. Os questionários eram compostos por quinze perguntas, que visavam avaliar o que os prescritores pensam da 
utilização de plantas medicinais como tratamento, o que eles sabem sobre as plantas medicinais, o quanto suas formações acadêmicas contribuíram para isso e qual o grau de interesse no assunto.

\section{RESULTADOS E DISCUSSÃO}

Todos os médicos e enfermeiros que trabalhavam na UBS na época da pesquisa aceitaram participar da pesquisa e responderam ao questionário. Quando perguntados se prescrevem ou indicam plantas medicinais para os usuários da UBS, a maioria, tanto dos médicos quanto dos enfermeiros, respondeu que prescreve eventualmente, como podemos observar nas Figuras 1 e 2.

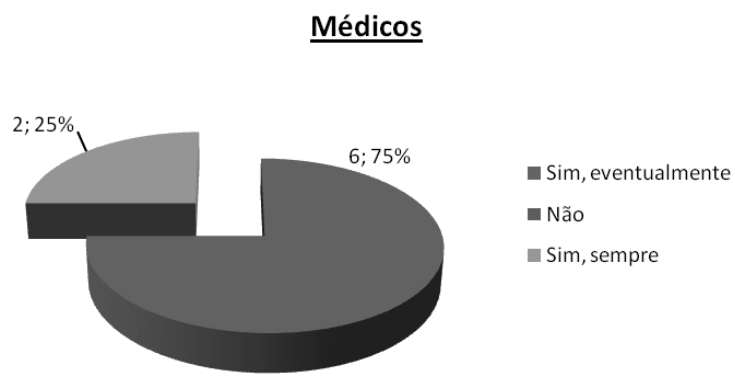

Figura 1: Frequência com que médicos prescrevem plantas medicinais aos usuários da UBS.

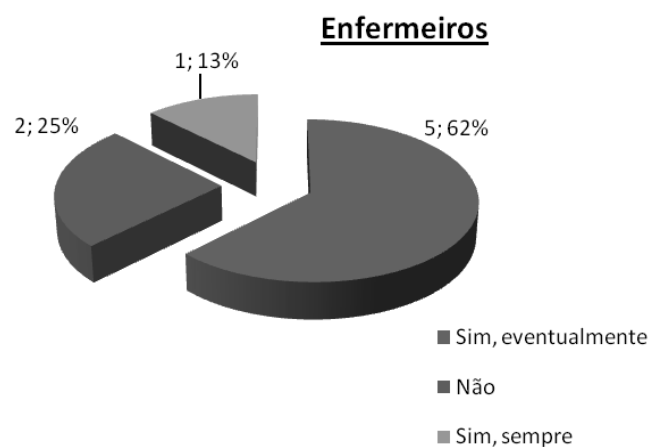

Figura 2: Frequência com que enfermeiros indicam o uso de plantas medicinais pelos usuários da UBS.
Os dados foram coletados em novembro de 2010 e depois foram tabulados e analisados.

O uso de fitoterápicos como antidepressivo/calmante foi o mais citado, o que também foi observado no estudo de Rosa et al. (2011), seguido pelos usos para tosse, gripe e mal estar gástrico, foram os mais citados pelos participantes da pesquisa. Na tabela 1 encontramos a lista completa das situações para os quais os entrevistados prescrevem/indicam plantas medicinais.

O Sistema Único de Saúde (SUS), no período de realização da pesquisa, oferecia à população oito medicamentos fitoterápicos, sendo eles, a alcachofra, aroeira, cáscara sagrada, garra do diabo, isoflavona, guaco e espinheira santa; além desses, o município também oferecia o extrato de valeriana. 0 que podemos observar é que os usos mais citados são aqueles para os quais a própria UBS fornecia os fitoterápicos, por exemplo, o extrato de valeriana, que é um antidepressivo/calmante e o xarope de guaco, utilizado para tosse.

Entre aqueles que disseram que prescrevem ou indicam plantas medicinais $(n=14)$, quando perguntados como consideravam o resultado dos tratamentos para os quais foram prescritos plantas medicinais ou fitoterápicos, 13 (93\%) consideraram satisfatórios e apenas 1 (7\%) considerou não satisfatório.

Dentre todos os entrevistados, apenas um afirmou ter tido contato com plantas medicinais durante a formação acadêmica, e isso é explicado, por este médico ter uma especialização em fitoterapia. Pois, durante 
a graduação este também não teve contato, assim como os outros colegas. A introdução de cursos sobre fitoterapia nos cursos de medicina é prática raríssima, apesar da inclusão de práticas complementares e integrativas nos currículos dos cursos de saúde ser sempre recomendada (QUEIROZ, 2000). Dessa forma, esses profissionais acabam sendo forçados a procurar embasamento teórico e científico sobre o assunto ao se deparar com a demanda dos próprios pacientes já durante a prática profissional.

Tabela 1. Situações em que os entrevistados prescrevem/indicam plantas medicinais ou fitoterápicos.

\begin{tabular}{|c|c|c|}
\hline \multirow[t]{2}{*}{ Situação } & \multicolumn{2}{|c|}{ Número de Citações } \\
\hline & Médico & Enfermeiros \\
\hline Antidepressivo/calmante & 8 & 1 \\
\hline Tosse & 5 & 1 \\
\hline Gripe & 4 & 3 \\
\hline Mal estar gástrico & 3 & 2 \\
\hline Sistema Respiratório & 2 & 2 \\
\hline Emagrecimento & 2 & - \\
\hline Hipertensão & 2 & 2 \\
\hline Problemas intestinais & 2 & 2 \\
\hline Diabetes & 2 & 1 \\
\hline Sistema geniturinário & 2 & 1 \\
\hline Pele & 1 & 2 \\
\hline Menopausa & 1 & 1 \\
\hline Placebo & 1 & - \\
\hline Doenças infecciosas/parasitárias & 1 & - \\
\hline Doenças osteomusculares & 1 & - \\
\hline
\end{tabular}

Em decorrência do pouco conhecimento, 94\% dos entrevistados disseram ter interesse em conhecer e aprender mais sobre plantas medicinais, uma vez que, a maioria não sente segurança em prescre- ver esse tipo de medicamento justamente em decorrência da falta de informações sobre as plantas, seus efeitos colaterais, interações com outros medicamentos e toxicidade. E também disseram que com 
um maior conhecimento e caso mais medicamentos feitos a partir de plantas medicinais fossem disponibilizados para as UBS, a prescrição poderia ser maior. É fato constante, observado também em outros estudos, que os profissionais sentem a necessi- dade de confirmação da eficácia dos medicamentos fitoterápicos e das plantas medicinais por meio de estudos científicos in vitro e in vivo, para poderem depositar maior confiança neste tipo de medicamento (ROSA et al., 2011).

\section{CONCLUSÕES}

A partir dos resultados, podemos concluir que boa parte dos entrevistados prescreve plantas medicinais. Porém, a maioria não teve contato com o assunto durante a formação, e muitas vezes por falta de conhecimento a respeito de atividade farmacológica, efeitos adversos, interações com outros medicamentos e toxicidade de plantas, ficam com receio de prescrever plantas medicinais. Além disso, os cursos de graduação não estão dando muita atenção a fitoterapia e terapias complementares em suas grades curriculares, o que faz com que os profissionais se deparem com a demanda no exercício da prática profissional e não saibam muito bem como agir.

Dessa forma, o ideal é que esses profissionais sejam treinados, bem informados e capacitados a respeito do assunto para que possam prescrever mais plantas medicinais aos usuários da UBS, o que é bom, pois além destes medicamentos serem mais baratos, também são bem aceitos pela população. Além disso, com mais informação, eles poderiam informar melhor estes usuários e evitar vários problemas decorrentes da automedicação.

\section{REFERÊNCIAS}

BRASIL. Ministério da Saúde. Portaria n 971, de 3 de maio de 2006. Aprova a Política Nacional de Práticas Integrativas e Complementares no Sistema Único de Saúde. Diário Oficial da União, n. 84, seção 1, 2006. 19p.

BRASILEIRO, B.G.; PIZZIOLO, V.R.; MATOS, D.S.; GERMANO, A.M., JAMAL, C.M. Plantas medicinais utilizadas pela população atendida no "Programa de Saúde da Família”, Governador Valadares, MG, Brasil. Revista Brasileira de Ciências Farmacêuticas, v. 44, n. 4, p. 629-636, 2008.

CARVALHO, M.C.G.; PIRES, R.L.; FLORINDO, W.S.; CAVALCANTI, A.S.S. Evidências para o uso de Indigo naturalis no tratamento da psoríase tipo placa: uma revisão sistemática. Natureza on line, v. 8, n. 3, p. 127-131, 2010.
MOREIRA, R.C.T.; COSTA, L.C.B.; COSTA, R.C.S.; ROCHA, E.A. Abordagem Etnobotânica acerca do Uso de Plantas Medicinais na Vila Cachoeira, Ilhéus, Bahua, Brasil. Acta Farm. Bonaerense, v. 21, n. 3, p. 205-211, 2002.

ORGANIZAÇÃO MUNDIAL DA SAÚdE. Cuidados Primários em Saúde. Relatório da Conferência Internacional sobre Cuidados Primários da Saúde, Alma Ata, URSS, 6 a 12 de setembro de 1978. Brasília: Ministério da Saúde, 1979. 64p.

QUEIROZ, M.S. O itinerário rumo às medicinas alternativas: uma análise em representações sociais de profissionais da saúde. Cad Saude Publica, v. 16, n. 2, p. 363-375, 2000. 
ROSA, C.; CÂMARA, S.C.; BÉRIA, J.U. Representações e intenção de uso da fitoterapia na atenção básica à saúde. Ciência \& Saúde Coletiva, v. 16, n. 1, p. 311318, 2011.

SCHENKEL, E.P.; SIMÕES, C.M.O.; MENGUE, S.S.; MENTZ, L.A.; IRGANG, B.E.; STEHMANN, J.R. O espaço das plantas medicinais e suas formas derivadas na medicina científica. Caderno de Farmácia, v.1, n. 2, p. 65-72, 1985.

Recebido em: 11 de junho de 2012 Avaliado em: 25 de julho de 2012 Aceito em: 10 de agosto de 2012
TOMAZZONI, M.I.; NEGRELLE, R.R.B.; CENTA, M.L. Fitoterapia popular: A busca instrumental enquanto prática terapêutica. Texto Contexto Enferm, v. 15, n. 1, p. 115-121, 2006.

VEIGA JUNIOR, V.F. Estudo do consumo de plantas medicinais na Região Centro-Norte do Estado do Rio de Janeiro: aceitação pelos profissionais de saúde e modo de uso pela população. Revista Brasileira de Farmacognosia, v. 18, n. 2, p. 308-313, 2008.

\footnotetext{
Mestranda do Programa de Pós Graduação em Biociências Aplicadas à Farmácia - Universidade Estadual de Maringá - UEM, Maringá - Paraná. E-mail: lopes.a.mariana@gmail.com.

2 Farmacêutica da Unidade Básica de Saúde Pinheiros - Maringá, Paraná. E-mail: simoniobici@yahoo.com.br

3 Doutora em Ciências Biológicas. Docente da Universidade Estadual de Maringá - UEM, Maringá - Paraná. E-mail: almalbiero@uem.br
} 